\section{Hörverlust bei Älteren erschwert die Kommunikation}

\section{Jeder vierte Ältere über 60 hat einen Hörverlust von mindestens $25 \mathrm{~dB}$ und} bei den über 70-Jährigen betrifft dies bereits jeden zweiten. Medizinische Irrtümer passieren häufiger bei Älteren und hinter diesen stecken oft Kommunikationsfehler, wie eine irische Studie zeigt.

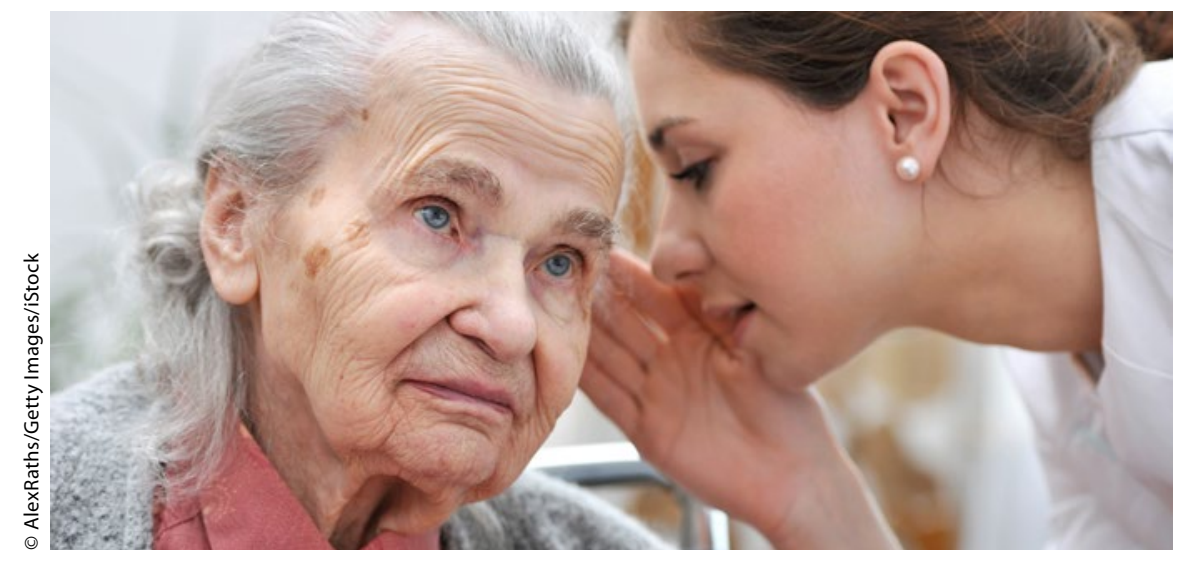

n einer aktuellen Studie untersuchten Vikki Cudmore et al. an der irischen University Cork die Kommunikationsprobleme Älterer in medizinischen Settings wie Arztpraxen und Ambulanzen. Aufgenommen wurden in die nicht repräsentative Stichprobe 100 ambulante Patienten im mittleren Alter von 73 Jahren an der Universitätsklinik Cork (Irland). 21 hatten einen unilateralen und 36 einen bilateralen Hörverlust. Nur eine Minderheit von 41 war nicht oder nur geringfügig beeinträchtigt $(<25 \mathrm{~dB})$. Nur jeder zweite hatte sich bereits einer Audiometrie unterzogen, 26 trugen ein Hörgerät. Die Audiometrie ist eine zuverlässige Methode zur Diagnose von Hörverlusten, allerdings haben nicht alle Patienten mit vergleichbaren Tonschwellen und Hörbefunden die gleichen Verständnisschwierigkeiten. In der aktuellen Studie wurde mit einem halbstrukturiertem Interview detaillierte Daten zur Prävalenz von Hörverlusten und den besonderen Inhalten und Problemen der Kommunikation erhoben und qualitativ analysiert.

43 Befragte klagten, dass sie den Arzt oder Pflegerin während der Konsultation oder dem Spitalaufenthalt nicht richtig verstanden hatten. Besonders unverständlich waren krankheits- oder
Aussprache, der Betonung und ähnlich klingenden Wörtern, die das Verstehen erschwerten. Eine weitere Hürde war die selektive Taubheit bei vier Betroffenen, die sich lieber taub stellten: „Wenn du etwas nicht hören willst, musst du auch nicht zuhören." Bei zwei Patienten scheiterte das Gespräch an dem Lärm im Untersuchungszimmer, da sie andere Stimmen nicht ausblenden konnten. Mehrfachnennungen waren möglich.

\section{Kommunikationsbarrieren abbauen}

Viele Ältere leiden an Hörverlusten, aber auch die Umstände der Konsultation beeinflussen das Verstehen. Störende Hintergrundgeräusche, die Redegeschwindigkeit und die Wortwahl ohne Fachjargon sowie eine klare, langsame Aussprache sind ebenso wie die gesamte psychosoziale Ausgangssituation (Furcht vor einer Krankheit) zu berücksichtigen. Hörgeräte alleine können die komplexen Kommunikationshürden nicht beseitigen. Der altersbedingte Hörverlust wirkt sich negativ auf die klinische Kommunikation aus. Die Autoren empfehlen dem medizinischen Personal, die Kommunikation in Inhalt und Form sowie der Umgebung vermehrt auf diese Patientengruppe auszurichten.

Stephanie Mühlbauer gehört hatten. 16 Patienten gaben an, dass die Kommunikation zu leise, zu schnell und durch zu viele Störfaktoren wie Stimmen so stark behindert war, dass sie dem Inhalt nicht folgen konnten. Sechs (10\%) Befragte scheiterten an der
Cudmore V, et al. Age-Related Hearing Loss and Communication Breakdown in the Clinical Setting. JAMA Otolaryngol Head Neck Surg. 2017 Aug 24. doi: 10.1001/jamaoto.2017.1248. 